\title{
Cardiac myosin-binding protein C: how a novel biomarker could transform chest pain triage
}

\author{
Thomas E Kaier*,1, Bashir Alaour ${ }^{1}$ \& Michael Marber ${ }^{1}$ \\ ${ }^{1}$ King's College London BHF Centre, The Rayne Institute, St Thomas' Hospital, London, UK \\ *Author for correspondence: Tel.: + 440207188 1008; Fax: + 440207188 0970; tk@tomkaier.com
' $\mathrm{cMyC}$ might allow earlier rule-out of AMI and if the promise of more effective triage holds true up to a fifth of all patients could benefit from expedited discharge"

First draft submitted: 25 May 2018; Accepted for publication: 30 May 2018; Published online: 18 July 2018

Keywords: acute myocardial infarction $\bullet$ AMI $\bullet$ biomarkers $\bullet$ cardiac myosin-binding protein $C \bullet$ cardiac troponin $\bullet$ chest pain $\bullet \mathrm{CMyC} \bullet$ emergency department $\bullet$ triage

Chest pain is a common symptom - according to recent literature, it is responsible for at least $6 \%$ of all presentations to emergency departments - however, only about $10 \%$ of these patients have a final diagnosis of acute myocardial infarction (AMI) [1-5]. Chest pain triage is fraught with difficulties as physicians are increasingly caught at the interplay of sensitivity and specificity. Fewer patients now have the diagnostic electrocardiogram changes of ST elevation or depression that allow triage at presentation [6,7] - in fact, $68 \%$ of all patients eventually diagnosed with an acute coronary syndrome present with non-ST elevation myocardial infarction. [8]. Consequently, triage has become reliant on detection of elevated levels of the biomarker cardiac troponin (cTn) in the blood. This is enshrined in the universal definition of myocardial infarction [9] and guidelines published by the European Society of Cardiology (ESC) [10], mandating the detection of a cardiac biomarker rise and/or fall for the diagnosis of AMI.

\section{The challenging reality of chest pain triage}

The technological advances in evolving cTn assays to high-sensitivity tests comes at the expense of loss of specificity; as analyzers are increasingly able to provide quantifiable cTn levels in almost every individual [11,12]. The very definition of a hs-cTn assay - according to the International Federation of Clinical Chemistry and Laboratory Medicine Task Force on Clinical Applications of Cardiac Bio-Markers - includes a coefficient of variation $\leq 10 \%$ at the 99th centile value and the ability to measure at least $50 \%$ of healthy individuals with concentrations above the assay's limit of detection (LoD) [13,14]. The clinical reality of this advance in assay technology is that many more patients test 'troponin-positive', but not necessarily 'AMI-positive' - all in an attempt to overcome the limitations which made cTn inherently unsuited for early diagnosis of AMI: a slow rise and disappearance from the bloodstream after myocardial injury $[15,16]$. By the ESC's own admission, the clinical implications of using high-sensitivity (hs) cTn assays include a twofold increase of detection of type 2 AMI, approximately $20 \%$ relative increase in detection of type 1 AMI and "elevations up to threefold the upper reference limit may be associated with a broad spectrum of conditions."

\section{Sensitivity comes at a cost}

Emergency physicians are caught up in this sensitivity/specificity quagmire: they have to handle complex rulein/rule-out algorithms to optimize care for the patient with suspected acute coronary syndrome at the front door of the hospital [10,17]. Two aspects make this inherently more challenging: even with high-sensitivity assays, the ESC advocates a delay of $3 \mathrm{~h}$ after chest pain onset for the first blood draw to take place; many patients get caught up in an 'observe' zone of indeterminate risk - too high a cTn level for discharge, but too low to classify as AMI. Without doubt, evermore sensitive assays will establish a new reality of biomarker interpretation in acute medical services around the world - the (nearly) always-quantifiable level of a cardiac biomarker ought to be scrutinized in

Future $\because$ Medicine 
the context of the clinical presentation, and we can no longer rely on an outdated black and white approach. But maybe we can achieve more effective triage using a highly sensitive and specific biomarker with a more favorable release profile.

\section{There might be another way}

Cardiac myosin-binding protein $\mathrm{C}(\mathrm{cMyC})$ is a promising novel biomarker of myocardial injury - originally described as the C-protein by Offer $e$ al. in 1973 [18], its discovery relied on the characterization of 'impurities' detected alongside myosin. $\mathrm{cMyC}$ has distinctive release kinetics that should enable it to act as a better adjudicator of acute versus chronic myocardial injury than troponin [19]. We have raised monoclonal antibodies targeting the cardiac isoform of myosin-binding protein $\mathrm{C}$ and successfully migrated the assay onto a high-sensitivity platform [12]. Subsequently, we demonstrated up to tenfold greater abundance of $\mathrm{cMyC}$ after myocardial injury than two leading hs-cTnT/I assays [20]. In a small study, involving 174 patients presenting within $3 \mathrm{~h}$ of chest pain onset and suspected AMI, we demonstrated a more dynamic rise of cMyC in the early stages of AMI than hs-cTnI [21]. This faster rise ought to yield a positive result (for rule-in of AMI), or an earlier reliable negative result (for rule-out of AMI). Furthermore, the relative abundance of $\mathrm{cMyC}$ should allow careful calibration of rule-out and rule-in thresholds, with more 'headroom' to enable precise quantification at the low concentrations needed for rule-out.

\section{Large chest pain study confirms efficacy}

This hypothesis was tested in a study of $>1900$ patients with symptoms suggestive of AMI - in an internal derivation/validation split, we derived cut-off thresholds for immediate rule-out or rule-in of AMI using cMyC instead of hs-cTnT/I (modeled on the 2015 ESC non-ST elevation myocardial infarction guideline) [10,22]. At similar diagnostic accuracy (based on comparable area under the receiver-operating characteristics curve), cMyC was substantially more effective than either hs-cTn assay in guiding patients to (safe) rule-out or rule-in: the net reclassification improvement demonstrated $14.9-23.5 \%$ better triage efficiency, thus reducing the size of the 'observe' zone substantially. In an institution such as St Thomas' Hospital, a central London hospital home to a tertiary cardiac unit, about 7800 patients are subject to hs-cTnT testing in the emergency department annually [23]. Our findings would translate into savings of 1000 bed-days per year - simply by achieving a more effective triage with a single blood draw at presentation.

\section{Faster \& better point-of-care}

But, there is an even greater goal to aim for: point-of-care testing (POCT) of cardiac biomarkers. To date, there is no POCT device that can achieve the levels of sensitivity required to provide accurate measurement of troponin for rule-out of AMI. This task requires a POCT assay to achieve a limit of detection equivalent to the laboratory assay, as the ESC guidelines advocate rule-out only in patients with undetectable hs-cTn levels. The best cTnT-POCT platform (Roche Cobas h323-handheld instrument) can detect a laboratory-equivalent value of $50 \mathrm{ng} / \mathrm{l}$ - about 3.5-fold greater than the 99th centile, or tenfold the LoD of the laboratory assay [24]. While not bioequivalent, it is tempting to speculate whether $\mathrm{cMyC}$ - with a tenfold greater abundance, and a rule-out threshold 25 -fold the LoD of the current laboratory platform - might facilitate easier migration onto a handheld device. This would allow rapid deployment of a novel cardiac biomarker to secondary and tertiary care facilities, and pave the way to a cluster-randomized controlled trial. Such a trial, with ethical consent at institutional level to ensure rapid enrolment of a large number of participants, would allow - for the first time - a head-to-head comparison of the effectiveness of different cardiac biomarkers in acute chest pain triage. The potential advantages are compelling: as most patients presenting with chest pain do not have AMI, the goal must be to rule-out AMI in as many patients as possible at the earliest opportunity - in other words, with the first blood draw. Even better, if this could be facilitated in a prehospital setting, where $\mathrm{cMyC}$ seems to benefit from dynamic release kinetics [25]. $\mathrm{cMyC}$ might allow earlier rule-out of AMI [22] and if the promise of more effective triage holds true up to a fifth of all patients could benefit from expedited discharge or care where necessary.

In conclusion, $\mathrm{cMyC}$ is a cardiac-restricted protein which rapidly enters the systemic circulation after myocardial injury and is relatively more abundant than troponin. The biomarker performs favourably in the diagnosis of AMI and is particularly well-suited to a point-of-care diagnostic platform - which could transform the way we perform chest pain triage. 


\section{Author's contributions}

All authors have contributed to the manuscript and were involved at writing the final version.

\section{Financial \& competing interests disclosure}

The authors are supported by grants from the Medical Research Council (UK; G1000737), Guy's and St Thomas' Charity (R060701, R100404), British Heart Foundation (TG/15/1/31518, FS/15/13/31320) and the UK Department of Health through the NIH Research Biomedical Research Centre award to Guy's \& St Thomas' NHS Foundation Trust. M Marber is named as an inventor on a patent held by King's College London for the detection of $\mathrm{CMyC}$ as a biomarker of myocardial injury. The authors have no other relevant affiliations or financial involvement with any organization or entity with a financial interest in or financial conflict with the subject matter or materials discussed in the manuscript apart from those disclosed.

No writing assistance was utilized in the production of this manuscript.

\section{References}

1. Goodacre S. The health care burden of acute chest pain. Heart 91(2), 229-230 (2005).

2. Murphy NF. Hospital discharge rates for suspected acute coronary syndromes between 1990 and 2000: population-based analysis. BMJ 328(7453), 1413-1414 (2004).

3. Blatchford O, Capewell S, Murray S, Blatchford M. Emergency medical admissions in Glasgow: general practices vary despite adjustment for age, sex and deprivation. Br. J. Gen. Pract. 49(444), 551-554 (1999).

4. Harris T, McDonald K. Is the case-mix of patients who self-present to ED similar to general practice and other acute-care facilities? Emerg. Med. J. 31(12), 970-974 (2014).

5. Healthcare Quality Improvement Partnership. MINAP Analyses 2012. https://data.gov.uk/dataset/a517d8f2-cccd-4f8a-870e-4433bfc1d732/minap-analyses-2012

6. Jennings SM, Bennett K, Lonergan M, Shelley E. Trends in hospitalization for acute myocardial infarction in Ireland, $1997-2008$. Heart 98(17), 1285-1289 (2012).

7. Rogers WJ, Frederick PD, Stoehr E et al. Trends in presenting characteristics and hospital mortality among patients with ST elevation and non-ST elevation myocardial infarction in the National Registry of Myocardial Infarction from 1990 to 2006. Am. Heart J. 156(6), 1026-1034 (2008).

8. McManus DD, Gore J, Yarzebski J, Spencer F, Lessard D, Goldberg RJ. Recent trends in the incidence, treatment and outcomes of patients with STEMI and NSTEMI. Am. J. Med. 124(1), 40-47 (2011).

9. Thygesen K, Alpert JS, Jaffe AS et al. Third universal definition of myocardial infarction. Circulation 126(16), 2020-2035 (2012).

10. Roffi M, Patrono C, Collet J-P et al. 2015 ESC Guidelines for the management of acute coronary syndromes in patients presenting without persistent ST-segment elevation. Eur. Heart J. 37(3), 267-315 (2016).

11. Basuray A, French B, Ky B et al. Heart failure with recovered ejection fraction: clinical description, biomarkers and outcomes. Circulation 129(23), 2380-2387 (2014).

12. Marjot J, Liebetrau C, Goodson RJ et al. The development and application of a high-sensitivity immunoassay for cardiac myosin-binding protein C. Transl. Res. 170, 17-25 (2016).

13. Apple FS, Sandoval Y, Jaffe AS, Ordonez-Llanos J. Bio-Markers ITF on CA of C. Cardiac troponin assays: guide to understanding analytical characteristics and their impact on clinical care. Clin. Chem. 63(1), 73-81 (2017).

14. Apple FS, Jaffe AS, Collinson P et al. IFCC educational materials on selected analytical and clinical applications of high sensitivity cardiac troponin assays. Clin. Biochem. 48(4-5), 201-203 (2015).

15. Katus HA, Remppis A, Scheffold T, Diederich KW, Kuebler W. Intracellular compartmentation of cardiac troponin T and its release kinetics in patients with reperfused and nonreperfused myocardial infarction. Am. J. Cardiol. 67(16), 1360-1367 (1991).

16. Katus HA, Remppis A, Neumann FJ et al. Diagnostic efficiency of troponin T measurements in acute myocardial infarction. Circulation 83(3), 902-912 (1991).

17. Amsterdam EA, Wenger NK, Brindis RG et al. 2014 AHA/ACC Guideline for the management of patients with non-ST elevation acute coronary syndromes: a report of the American College of Cardiology/American Heart Association Task Force on practice guidelines. J. Am. Coll. Cardiol. 64(24), e139-e228 (2014).

18. Offer G, Moos C, Starr R. A new protein of the thick filaments of vertebrate skeletal myofibrils: extractions, purification and characterization. J. Mol. Biol. 74(4), 653-676 (1973).

19. Baker JO, Tyther R, Liebetrau C et al. Cardiac myosin-binding protein C: a potential early biomarker of myocardial injury. Basic Res. Cardiol. 110(3), 23 (2015).

20. Marjot J, Kaier TE, Martin ED et al. Quantifying the release of biomarkers of myocardial necrosis from cardiac myocytes and intact myocardium. Clin. Chem. 63(5), 990-996 (2017). 
21. Kaier TE, Anand A, Shah ASV, Mills NL, Marber M. Temporal relationship between cardiac myosin-binding protein C and cardiac troponin I in type 1 myocardial infarction. Clin. Chem. 62(8), 1153-1155 (2016).

22. Kaier TE, Twerenbold R, Puelacher C et al. Direct comparison of cardiac myosin-binding protein $\mathrm{C}$ with cardiac troponins for the early diagnosis of acute myocardial infarction. Circulation 136(16), 1495-1508 (2017).

23. Marjot J, Kaier TE, Henderson K, Hunter L, Marber MS, Perera D. A single centre prospective cohort study addressing the effect of a rule-in/rule-out troponin algorithm on routine clinical practice. Eur. Heart J. Acute Cardiovasc. Care doi:10.1177/2048872617746850 (2017) (Epub ahead of print).

24. Bertsch T, Chapelle J-P, Dempfle C-E, Giannitsis E, Schwabs M, Zerback R. Multicentre analytical evaluation of a new point-of-care system for the determination of cardiac and thromboembolic markers. Clin. Lab. 56(1-2), 37-49 (2010).

25. Kaier TE, Stengaard C, Marjot J et al. Cardiac myosin-binding protein $\mathrm{C}$ as alternative to cardiac troponin $\mathrm{T}$ for the diagnosis of acute myocardial infarction in the very early phase. J. Am. Coll. Cardiol. 69(11), 221 (2017). 